\title{
Quantitative assessment of the airway response to bronchial tests based on a spirometric curve shift
}

\author{
Adam G. Polak*, Member, IEEE, Andrzej Obojski, and Janusz Mroczka
}

\begin{abstract}
Objective: Although spirometry is the most common pulmonary function test, there is no method to quantitatively infer about airway resistance or other properties from the flow-volume curves. Recently, an identifiable inverse model for forced expiration was proposed, as well as the idea to deduce changes in airway resistances and compliances from spirometric curve evolution. The aim of this work was to combine the above advances in a method for assessing the airway response to bronchial tests from a spirometric curve shift. Methods: The approach is based on the differential measurement of the degree, site of maximal effect and width of changes, further recalculated into relative changes in the distribution of airway resistances $\left(\delta R_{g}\right)$ and compliances $\left(\delta C_{g}\right)$ along the bronchial tree. To this end, appropriate models were identified using the pre- and post-test spirometry data. The accuracy was validated using sets of data simulated by the anatomy and physiology based models. Finally, the method was used to analyze the bronchodilation tests of three asthmatic subjects. Results: The expected errors in assessing the degree, site and width of changes in the zone of conducting airways were $6.3 \%$, 2.4 generations and $22 \%$, respectively, and for $\delta R_{g}$ and $\delta C_{g}$ were $5-10 \%$ and $13-16 \%$, respectively. The analyses of clinical data indicated a significant reduction in resistances and an increase in compliances of airway generations 8-12, consistent with clinical knowledge. Conclusion: An unprecedented method to plausibly transforming the spirometry data into the site and degree of changes in airway properties has been proposed. Significance: The method can be used to deduce about the effects of bronchial tests, as well as to monitor changes in the airways between visits or to investigate how inhaled pharmaceuticals affect the bronchi.
\end{abstract}

Index Terms-Airway response, bronchial tests, differential measurement, model identification, spirometry.

\section{INTRODUCTION}

$\mathrm{S}$ PIROMETRY is part of the diagnosis and management of many respiratory diseases, including asthma and COPD [1][3]. It is often accompanied by bronchial tests consisting in the delivery of active inhalant compounds, affecting receptors in airway smooth muscle (ASM) [4], [5]. Depending on the agent, the airways dilate (bronchodilator tests) or constrict (provocation tests).

Manuscript accepted June 22, 2020, This work was supported by the National Science Centre, Poland, under Grant 2016/21/B/ST7/02233.

Copyright (c) 2020 IEEE. Personal use of this material is permitted. However, permission to use this material for any other purposes must be obtained from the IEEE by sending an email to pubs-permissions @ ieee.org.
The final effect is related, however, to several factors, such as: type of medicine, particles size, body position, inspiratory flow rate, airway geometry and dimensions related to age, as well as the concentration of ASM receptors [4], [6]-[12]. Nowadays, computational models involving fluid dynamics (CFD), supported by generic models for the bronchial tree or tomographic 3D scans, are typical tools for investigating this phenomenon. From those studies it follows that drug particles deposit within successive bronchial tree generations, rather non-uniformly, more efficiently in the lower than upper airways in adults, more intensively on walls exposed to airflow, and in airway bifurcations [7], [10], [12], however showing a roughly monomodal distribution along the bronchial tree [8], [9]. The effects of bronchial tests are evaluated by spirometry, particularly by analyzing changes in spirometric parameters, such as the forced expiratory volume in $1 \mathrm{~s}$ (FEV1) and/or the forced vital capacity (FVC), in reference to baseline or predicted values [1]. These global indices are not specifically related, however, to the site and degree of mechanical changes along the bronchial tree, although such outcomes would be of great clinical interest [13], [14]. Undoubtedly, more information about the actual airway response to the inhaled particles is included in the whole spirometric curve (forced expiratory airflow plotted against the expired volume), and even more in a spirometric curve shift caused by the test.

Quantitative inference about lung mechanics from a spirometric curve is potentially possible by fitting an appropriate mathematical model to spirometry data. Modeling the respiratory system has a long history, nevertheless, most of the morphology- and physiology-based approaches are lumpedparameter or fluid-dynamics models assuming stiff airway walls [15]-[20]. As such, they are not suitable to properly capture distributed phenomena in flexible airways, crucial for the forced expiration process. Despite these difficulties, the computational model for forced expiration has been proposed and widely recognized [21], and then successfully further developed [22]-[24].

Unfortunately, the identification of that model proved to be a great challenge due to a large number of parameters, their co-

*A. G. Polak and J. Mroczka are with the Department of Electronic and Photonic Metrology, Wrocław University of Science and Technology, Wrocław, Poland (correspondence e-mail: adam.polak@pwr.edu.pl). A. Obojski is with the Department of Internal Medicine and Allergology, Wrocław Medical University, Wrocław, Poland. 
influences on the output and model nonlinearities [25]. To overcome these problems, only one property of the airways (their maximal areas in the first 17 generations) was estimated in the first trial - initially by manual manipulation [26], and then using the simulated annealing algorithm [27]. In a more extensive approach, two parameters of the airways were changed to reproduce a lung function evolution after heart-lung transplantation [28]. Though the model could be fitted to data considerably well in these studies, omitting the estimation of other influential parameters has decreased the reliability of this method and prevented its practical application. Just recently, a new approach to the identification of the forced expiration model was proposed, together with the idea how to translate estimated parameter values into the distributions of airway resistances and compliances along the bronchial tree [29]. The identified (inverse) model preserved the entire computational complexity, however, the number of estimated parameters was limited to 6 by applying a sophisticated procedure of model reduction. Moreover, recently it was also suggested, how to assess the effects of bronchodilation or provocation tests by fitting this model to pre- and post-test spirometry data [30]. Despite the evaluated errors of both parameter estimates (3.7\% to $16.6 \%$ in relation to their variability ranges) and the calculated airway resistances and compliances (7-35\% and 5$12 \%$, respectively) were rewardingly small [29], that preliminary study was done using merely synthetic data, generated with the model having the same computational structure and values of not estimated parameters as the inverse one.

Following those encouraging results, this work aims in proposing a new method for quantitative evaluation of the airway response to bronchial tests based on a spirometric curve shift in terms of airway generation resistances and compliances, as well as in validating it, combining the ideas from [29] and [30]. The novelty of this approach covers three main issues: the principle of differential measurement (suppressing the influence of uncertain model parameters) applied to assess the degree, site and width of response, as well as the relative changes in airway generation resistances and compliances, a new mathematical model for the modification of airway mechanics caused by a bronchial test, as well as using both synthetic and clinical data to evaluate the method. The synthetic data used for validating the method were generated by the inverse model [29], and, to avoid the inverse crime [31], by the computational models for forced expiration with full degrees of freedom: including the symmetrical [22] and asymmetrical bronchial tree [24]. Finally, the potential clinical application of this approach was illustrated using spirometry results from bronchodilation tests of three asthmatic patients.

\section{MAterials AND MethodS}

\section{A. Inverse Model for Forced Expiration (InvM)}

The inverse model used in this work has been presented in detail elsewhere [29] and will be briefly described here. Its computational scheme is the same as applied in the complex model for forced expiration through a symmetrical bronchial tree with 24 airway generation, with the difference that it has far fewer free parameters (6 instead of 154). First, the complex model parameters that have been found as insignificantly influential, are kept constant with values representing the normal lung (except the residual volume, RV, which is calculated as $150 \%$ of the predicted value for a given patient). Furthermore, the personalization of airway properties distribution along the bronchial tree for a given subject is achieved by rescaling their baseline properties (normal lung). Particularly, the airway geometrical dimensions (lengths $l_{g}$ and maximal areas $A_{m}$ ) are calibrated using scalar values $p_{l}$ and $p_{l}^{2}$, and the distribution along the airway generations $g$ of two parameters characterizing the nonlinear dependence of airway area on transmural pressure $P_{t m}$ is modelled using the following sigmoid $k_{p}(g)$, with own parameters $p_{a 1}$ and $p_{a 2}$ :

$k_{p}(g)=\frac{0.0196 g+1.05}{1+\exp \left(p_{a 1} g+p_{a 2}\right)}-0.0174 g+0.7$

These parameters are: $\alpha_{0}$ (normalized area at $P_{t m}=0$ ) and $\alpha_{0}^{\prime}$ (slope of $\alpha_{0}$ at $P_{t m}=0$ ), rescaled with the proportion to $k_{p}(g)$ and $k_{p}{ }^{2}(g)$, respectively. This set $\left(p_{l}, p_{a 1}\right.$ and $\left.p_{a 2}\right)$ is completed with three other free parameters, describing the linear-exponential dependence of lung volume on transpulmonary pressure, i.e. the lung compliance $C_{s t}$ (from the linear part), $\Delta V_{0}$ and $\Delta V_{t r}$ (the differences between characteristic volumes and RV). Beside this, the InvM uses also patient-specific, easy determinable information: sex, age, height, and vital capacity (VC, a parameter of the original complex model).

\section{B. Model for the Airway Response to Bronchial Tests (MAR)}

The computational structure of the complex model is preserved also in the MAR. This time, however, all the lung properties used or estimated by the InvM are kept unchanged (so, the estimates of InvM parameters form the input together with the second spirometric curve), and only the changes in airway mechanics caused by a bronchial test are estimated.

Taking into account that the aerosol particles deposition has a generally monomodal distribution in the bronchial tree [8], [9], the effect of the bronchodilation or provocation test has been modeled by a Gaussian-like function:

$k_{\alpha}(g)=1-p_{s} \exp \left(\left(g-p_{g}\right)^{2} / p_{w}^{2}\right)$

where $p_{s}$ represents the degree of change, $p_{g}$ is the airway generation with maximal response, and $p_{w}$ describes the width of changes along the bronchial tree. With only 3 parameters, it is well-identifiable at the expense of intrinsic symmetry. Such a form of the MAR allows for mimicking both the monotonous distribution of response (increasing or decreasing) as well as changes concentrated in a limited region of airway generations (Fig 1). Thus, the values of $k_{\alpha}(g)$ are used to model the impact of a bronchial test only on the airway properties distribution, specifically by additionally scaling the previously assessed values of $\alpha_{0}$ and $\alpha_{0}^{\prime}$ by $k_{\alpha}(g)$ and $k_{\alpha}^{2}(g)$, respectively. 


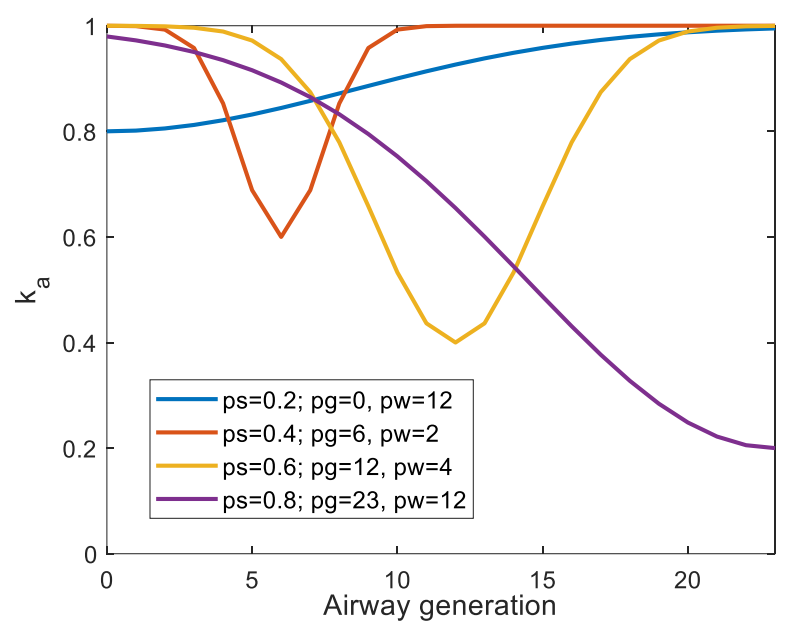

Fig. 1. Illustration of the potential of function $k_{\alpha}$ (for $p_{s}>0$ ) to scale airway properties along the bronchial tree.

\section{Estimation of Changes in Airway Mechanics}

All models, procedures and computations were implemented and executed in Matlab (R2017a, MathWorks). The overall scheme of data processing is depicted in Fig. 2. At the beginning, the descending parts of the pre- and post-test spirometric curves (determined by the well-defined flowlimiting mechanisms) are selected. Because the former sensitivity analysis of the InvM has revealed that the model is better identifiable for dilated airways [29], the analysis starts with the post-test data.

The parameters of the nonlinear InvM are estimated in two stages [29]. First, the global identification is performed using the inverse neural network (InvNN), and then the resulting rough estimates are used as the starting vector $\hat{\boldsymbol{\theta}}_{\text {start }}^{(6)}$ for the iteratively-minimized Levenberg-Marquardt procedure (LMIM). The final estimates $\hat{\boldsymbol{\theta}}_{I n v M}^{(6)}$ are obtained by solving the local optimization problem (minimizing the distance between the post-test spirometric curve and the model output). The estimation of MAR parameters is also done using LM-IM that fits this model to the pre-test spirometric curve, yielding $\hat{\boldsymbol{\theta}}_{M A R}^{(3)}$.

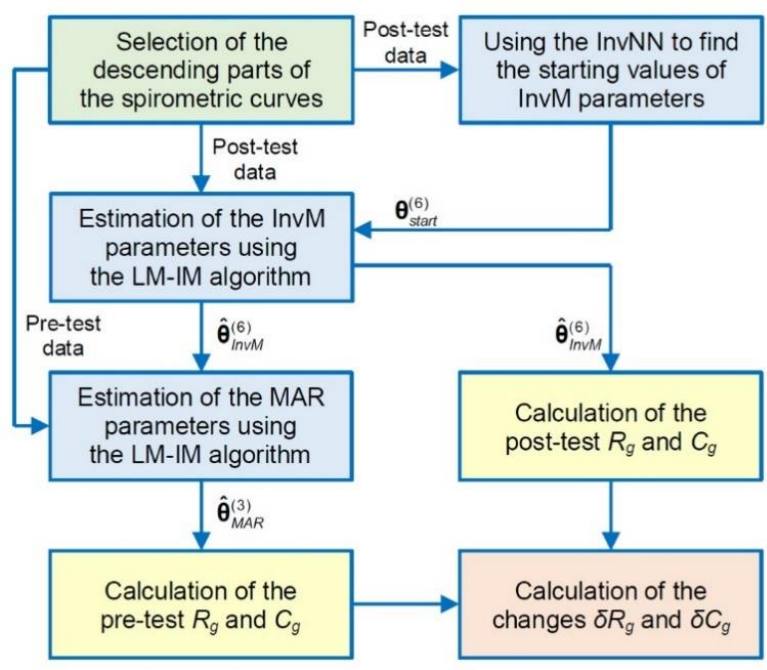

Fig. 2. The flowchart of the procedure.
The estimates achieved by analyzing the post- and pre-test spirometry data are used to calculate the values of mentioned earlier $\hat{A}_{m}, \hat{l}_{g}, \alpha_{0}$ and $\alpha_{0}^{\gamma}$ for each airway generation, specific for the analyzed state of a subject. Then, according to the idea presented in [29], these assessed airway parameters are recalculated into the clinically interpretable resistances and compliances of airway generations as follows:

$$
\begin{aligned}
& R_{g}(g)=\frac{a 8 \pi \mu l_{g}}{2^{g} A_{g}^{2}}=\frac{a 8 \pi \mu l_{g}}{2^{g} A_{m}^{2}\left(1-\left(1-\alpha_{0}\right)\left(1-P_{t m} / P_{2}\right)^{-n_{2}}\right)^{2}} \\
& C_{g}(g)=2^{g} l_{g} \frac{\partial A_{g}}{\partial P_{t m}}=2^{g} l_{g} A_{m}\left(\alpha_{0}-1\right) \frac{n_{2}}{P_{2}}\left(1-\frac{P_{t m}}{P_{2}}\right)^{-n_{2}-1}
\end{aligned}
$$

where $A_{g}$ is the current airway area, $P_{1}=n_{1} \alpha_{0} / \alpha_{0}^{\prime}, P_{2}=n_{2}\left(\alpha_{0}-\right.$ $1) / \alpha_{0}^{\prime}, a$ is an empirical coefficient, $\mu$ is the gas viscosity, $n_{1}$ and $n_{2}$ are the shape-adjusting exponents [21], and $P_{t m}=0.5 \mathrm{kPa}$ (typical value for the end of normal expiration).

The indices proposed in this work, characterizing the airway response and reflecting the undergoing physiological processes, are: $p_{s}, p_{g}, p_{w}$, and the relative changes of resistances $\left(\delta R_{g}\right)$ and compliances $\left(\delta C_{g}\right)$ in subsequent airway generations. Using general notation $\theta$ for $R_{g}$ and $C_{g}$, and ^ for parameter estimates, as well as subscripts pre and post for the values obtained from the pre- and post-test data, the estimated relative changes in airway properties are calculated for each of 24 airway generations as:

$\delta \hat{\theta}=\left(\hat{\theta}_{p o s t}-\hat{\theta}_{p r e}\right) / \hat{\theta}_{p r e} \cdot 100 \%$

After matching the models to the data, it is also possible to track the movement of the flow limiting site over generations during forced expiration [28], [32], and to find the flow-limiting generation with the smallest index. Only results representing this and upstream generations are further analyzed.

\section{Validation of the Method Using Synthetic Data}

Assessing the operation and accuracy of the proposed method, one needed the true values of airway properties underlying the analyzed shift in the spirometric curve. It was

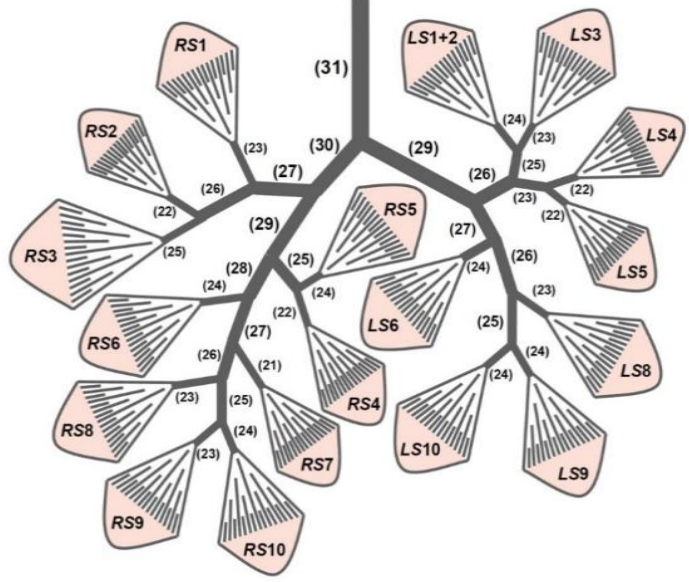

Fig. 3. Structure of the asymmetrical bronchial tree (airway orders in parentheses), $R S$ - ten right, and $L S$ - eight left bronchial segments. 
possible using spirometry data simulated by the computational models with known parameters. Three such models (described below) were applied in this work to generate 100 post-test curves by each. Having the post-test data, the corresponding 100 pre-test curves were simulated by randomly selecting the 3 parameters of MAR, with $p_{s}>0$ and $p_{g}$ within the intrathoracic bronchial tree. The first model was just the InvM with the difference that the full spirometric curves, not only the descending parts, were generated. In this case, different posttest flow-volume data were simulated by randomly changing 6 parameters of InvM within their ranges of variability (uniform distribution). The same computational scheme (referred to as SymM), including the symmetrical bronchial tree and the formerly drawn parameters for post- and pre-test cases, was then used to simulate another 100 pairs of curves, this time, however, the values of all other model parameters (kept constant so far) were randomly drawn from the assessed ranges of their variability. The third data set was generated analogously with the computational model mimicking the real lung most closely, i.e. including the asymmetrical bronchial tree (Fig. 3) - the AsymM [24]. All the synthetic data were finally disturbed by additive white noise $(\mathrm{SD}=0.01 \mathrm{~L} / \mathrm{s})$.

The advantage of using synthetic data is that the true parameter values of the models used to generate them are known. Thus, by analogy to (5) and using superscripts * to denote the true values of airway resistances and compliances, one can calculate the true changes in airway mechanics that underlie the shifts in simulated and then analyzed spirometric curves:

$$
\delta \theta^{*}=\left(\theta_{p o s t}^{*}-\theta_{p r e}^{*}\right) / \theta_{p r e}^{*} \cdot 100 \%
$$

Finally, knowing the estimated and true indices of airway response to the bronchial test in the $i$ th case, it is possible to calculate the error of the proposed method:

$$
\Delta_{\delta \theta}(i)=\delta \hat{\theta}_{i}-\delta \theta_{i}^{*}
$$

The above operation is straightforward for the analyzes done with data generated by the InvM and SymM, which include 24 airway generations. On the contrary, the AsymM (Fig. 3) incorporates the structure with 31 airway orders $($ ord $)$. To translate the true values of resistances and compliances computed for the 31 airway orders of the asymmetrical model into 24 values necessary to compare $\delta \theta^{\top}$ with $\delta \theta^{*}$, the linear mapping $g=-23 / 30 \times$ ord $+713 / 30$ was used to resample $R^{*}$ (ord) and $C^{*}$ (ord) into $R_{g}^{*}$ and $C^{*}{ }_{g}$ before computing (6). In addition, the percentage of airway orders with the maximal response, correctly indicated by the estimated generations $p_{g}$, was assessed for this case, using the transformation table given in [35].

Having 100 evaluations of $\Delta_{\delta \theta}$ for each set of data, the expected difference between the estimated and true airway responses were computed in $L_{2}$ as the root-mean-square errors for each airway generation [33]:
$\operatorname{RMSE}_{\delta \theta}=E\left\|\delta \hat{\theta}-\delta \theta^{*}\right\|_{2} \cong \sqrt{\frac{1}{100} \sum_{i=1}^{100} \Delta_{\delta \theta}^{2}(i)}$

RMSE was also calculated for the estimates of $p_{s}, p_{g}$ and $p_{w}$, separately for the conducting airways (generations $0-16$ ) and the acinar zone (generations 17-23).

\section{E. Assessment of Airway Response Using Clinical Data}

To test the effectiveness of the proposed method in clinical settings, spirometry data from the bronchodilator tests of three male asthmatic patients (\#1: height $170 \mathrm{~cm}$, age 22 years; \#2: $158 \mathrm{~cm}, 43$ years; \#3: $180 \mathrm{~cm}, 27$ years), were analyzed. The study protocol had been approved by the Bioethics Committee of the Wrocław Medical University. Informed consent was obtained from all subjects before and study procedure was performed. The measurements were done using a PC-based Penumotrac Spirometer (Vitalograph). All patients were recruited during routine physician office visits. The lung function test protocols used in the study were in accordance with the ATS/ERS recommendations [1], [5]. In particular: i) three acceptable and reproducible slow vital capacity (VC) tests were performed and followed by at least three acceptable and reproducible forced expiratory maneuvers, ii) the bronchodilator (salbutamol MDI, four $100 \mathrm{mcg}$ actuations) was inhaled, iii) three acceptable and reproducible slow and forced maneuvers were recorded again $15 \mathrm{~min}$ after bronchodilator intake. After the tests, depersonalized spirometry data were exported from Spirotrac Software (Vitalograph) to .csv files that were finally downloaded into Matlab. Patient's sex, height, age and VC are external parameters of the InvM and MAR. Due to between-maneuver variability, the largest measured VC $\left(\mathrm{VC}_{\max }\right)$ for a given subject was used as the model input. If the $\mathrm{VC}_{\max }$ was smaller than the biggest $\mathrm{FVC}$, the input value of $\mathrm{VC}$ was set to $1.01 \times \mathrm{FVC}$ ( $\mathrm{VC} \geq \mathrm{FVC}$ according to physiology, so this is assumed in the models). Having three pre- and post-test spirometric curves, the first volume from the descending and concave arms was found (the largest value form the 3 curves), then these parts were evenly resampled using splines at 100 points in the range to $\mathrm{VC}$, and finally averaged. The InvM and MAR were then fitted to the mean descending post- and pre-test data, yielding the estimates used to assess the airway response to the test.

\section{RESULTS}

The models InvM and MAR were fitted to all the post-and pre-test spirometric curves from the three generated sets, yielding relevant estimates $\hat{\boldsymbol{\theta}}_{I n v M}^{(6)}$ and $\hat{\boldsymbol{\theta}}_{M A R}^{(3)}$. The fits were good, even in cases of large differences between the final estimates and true parameter values of MAR (Fig. 4).

The most important outcome of this study was the evaluation of the proposed method accuracy. Table I summarizes the expected errors of estimating the MAR parameters (to facilitate interpretation, the errors of response intensity and width are presented in relation to their true values, however the error of location of maximal response as the absolute number of 


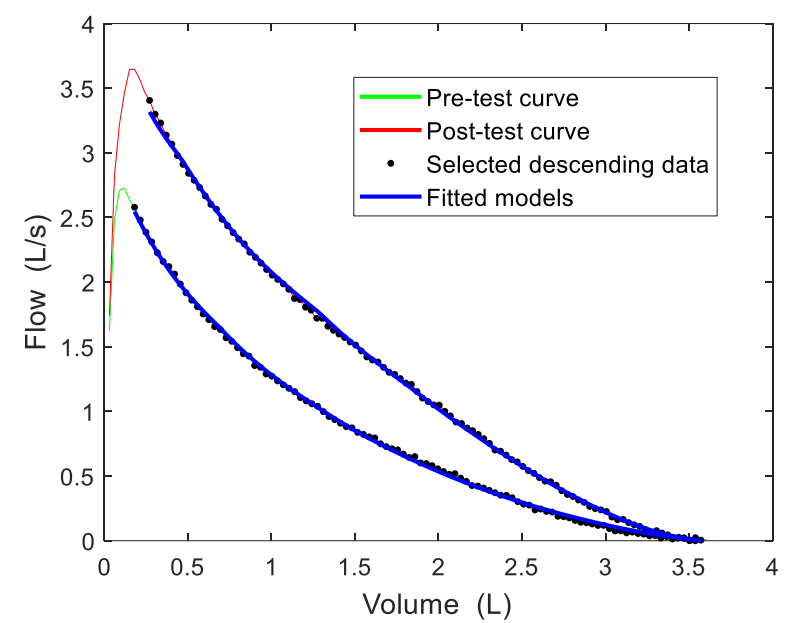

Fig. 4. Fits of the models to the synthetic data generated using the asymmetrical bronchial tree (the case of least accurate estimation of the MAR parameters).

generations). The intensity $\left(p_{s}\right)$ and generation with maximal response $\left(p_{g}\right)$ were assessed more accurately for the conducting bronchi, but the width of response $\left(p_{w}\right)$ for the acinar airways. All estimation errors increased, comparing the use of data from the InvM, SymM and AsymM models. Additionally, analyzing the results of MAR identification from the AsymM data, the percentage of airway orders with the maximal response, correctly indicated by the estimated $p_{g}$, was $91,5 \%$ and $78.1 \%$, if $p_{g}$ belonged to the zone of conducting or acinar airways, respectively. Also according to Fig. 5, the best accuracy was achieved when analyzing the data simulated by the InvM, and the least precision for the data generated by the AsymM. It turned out that in this worst case, the assessment of resistance and compliance changes along the conducting airways (generations 0-16) was more accurate (expected errors at the level of $5-10 \%$ and $13-16 \%$, respectively) than in the range of acinar airways (generations 17-23), where the errors grew towards the lung periphery up to 20 and $32 \%$, respectively. Flow limiting generations need not be the same in the narrowed and dilated airways, so the above evaluation was shown beginning with the $2^{\text {nd }}$ or $3^{\text {rd }}$ generation, since the site of flow limitation, tracked during estimations, did never appear in the trachea or main bronchi in both pre- and post-test cases.

TABLE I

ACCURACY (RMSE) OF THE ASSESSED INTENSITY $\left(P_{S}\right)$, SITE $\left(P_{G}\right)$ AND WIDTH $\left(P_{W}\right)$ OF THE AIRWAY RESPONSES TO BRONCHIAL TESTS

\begin{tabular}{|c|c|c|c|c|c|c|}
\hline \multirow{3}{*}{ Source of data } & \multicolumn{6}{|c|}{ RMSE } \\
\hline & \multicolumn{3}{|c|}{ Conducting airways } & \multicolumn{3}{|c|}{ Acinar airways } \\
\hline & $p_{s}(\%)$ & $p_{g}($ gen $)$ & $p_{w}(\%)$ & $p_{s}(\%)$ & $p_{g}$ (gen) & $p_{w}(\%)$ \\
\hline InvM & 3.5 & 1.1 & 18.5 & 10.9 & 2.4 & 9.9 \\
\hline SymM & 6.3 & 2.4 & 21.9 & 16.0 & 3.6 & 20.3 \\
\hline AsymM $^{\text {a }}$ & 15.9 & 3.9 & 42.1 & 34.8 & 5.3 & 30.2 \\
\hline
\end{tabular}

TABLE II

ASSESSED INTENSITY $\left(P_{S}\right)$, SITE $\left(P_{G}\right)$ AND WIDTH $\left(P_{W}\right)$ OF THE AIRWAY RESPONSES TO BRONCHIAL TESTS

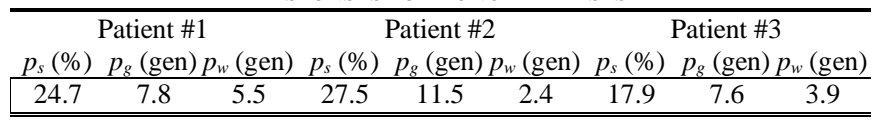
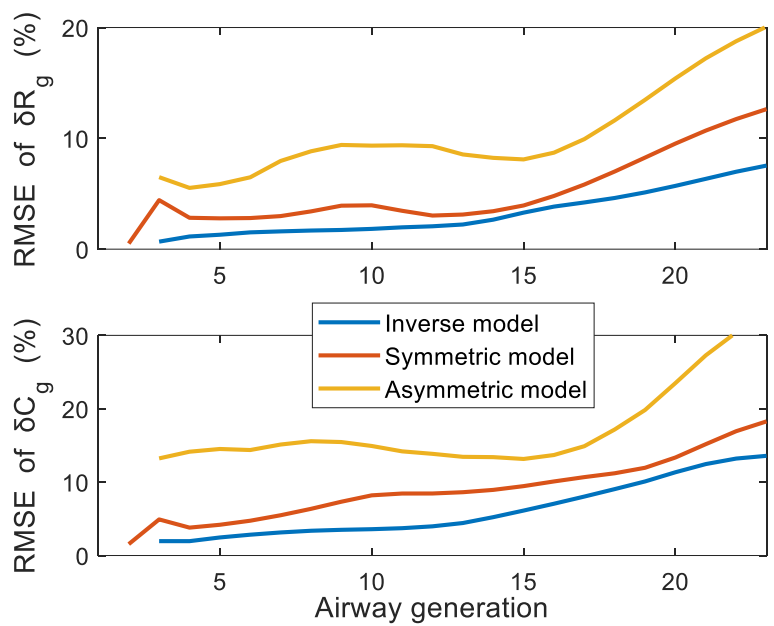

Fig. 5. Accuracy (RMSE) of the method assessed according to (8) and using the synthetic data generated by the inverse (InvM), symmetrical (SymM) and asymmetrical (AsymM) models.

In addition, the proposed method was used to analyze real spirometry data from the bronchodilation tests of three asthmatic subjects. Also in these cases, the models matched the data well. An example of such results is illustrated in Fig. 6, where the three post- and pre-test spirometric curves are shown with the averaged data points of their descending parts and with the InvM and MAR fitted to these descending arms.

The final outcomes of the proposed method are shown in Tab II and Fig. 7. It is clearly visible, that the resistances have decreased to varying degrees (maximally 44-64\%) and compliances increased (34-64\%) in the zone of conducting airways. The responses to bronchodilation are concentrated around generation 8 for younger Patients \#1 and \#3 (involving mainly generations 5-10 and 6-10, respectively) and about generations 11-12 in the case of older Patient \#2 (involving mainly generation 10-13). Furthermore, the estimated response intensity $\left(p_{s}\right)$ is negatively correlated with patient height.

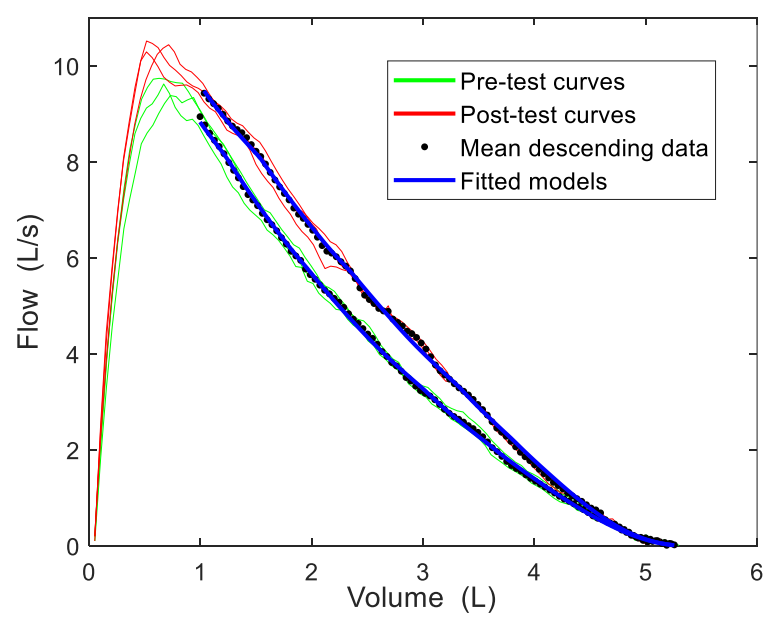

Fig. 6. Fits of the models to spirometry data from Patient \#3. 


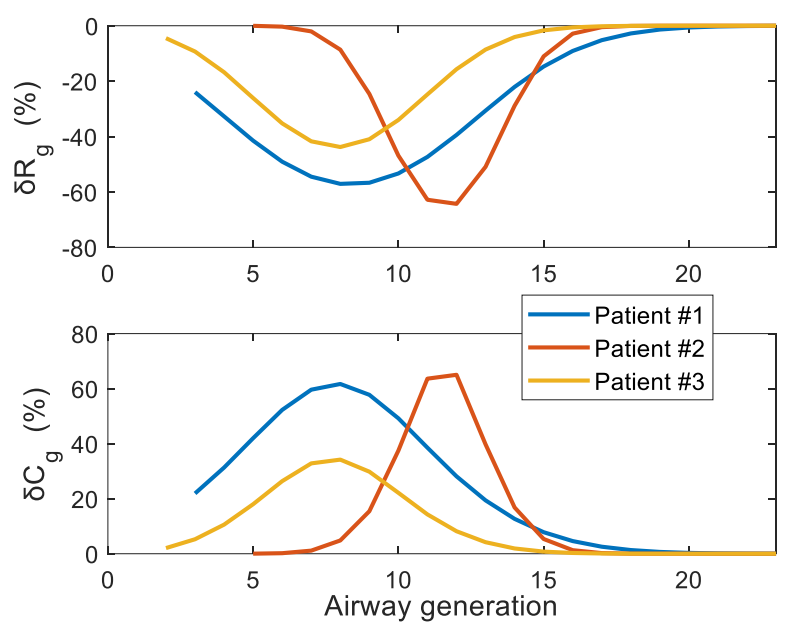

Fig. 7. Assessed responses of the airways to bronchodilation tests in terms of airway generation resistance and compliance changes $\left(\delta \hat{R}_{g}\right.$ and $\delta \hat{C}_{g}$, respectively).

\section{Discussion}

This work adopts several hypotheses and assumptions, justified at the time of their mention. Because they have an impact on the course and results of this research, the main ones are summarized here again: i) the symmetrical lung structure is sufficient to describe the effects of airway narrowing (particularly within higher generations) on the forced expiration curve; ii) the descending part of the curve can be sufficiently accurately described using the model with 6 free parameters; iii) the resulting shift of the spirometric curve after a bronchial test is mainly associated with the changes in airway mechanics; iv) airway narrowing causes interrelated changes in the relevant model parameters $\alpha_{0}$ and $\alpha_{0}^{\prime} ; \mathrm{v}$ ) the effect of inhaled aerosol is concentrated in a few neighboring airway generations and can be modelled by a Gaussian-like function with 3 free parameters.

The proposed method relies on determining changes in airway mechanics caused by a bronchial test from the shift of the spirometric curve, applying the principle of differential measurement. The main benefit of differential measurement is the reduction, and in some cases elimination of systematic errors resulting from imperfectly known, interfering quantities. In this method, representing indirect measurements consisting in the identification of mathematical models, the estimates of InvM that describe general properties of the lung (including the airways after the test), are kept constant when the MAR is identified. Even if the precision of InvM estimates is limited, the assessment of changes in airway mechanics, represented by the estimates of MAR parameters, is more accurate (see Appendix). The principle of differential measurement is also used in assessing changes in airway generation resistances and compliances (nominator in (5)). On the other hand, to provide easy interpretable quantities, the relative airway alternations are computed by dividing these differences by the more uncertain estimates $\theta_{\text {pre }}$. In effect, the relative airway responses are characterized by bigger errors then their absolute evaluations. This approach, however, is a conscious compromise between accuracy and enabling a graphical interpretation of the proposed method in terms of $\delta R_{g}$ and $\delta C_{g}$. In addition, because chronic respiratory diseases, such as asthma or COPD, are inherently heterogeneous [43], [44], the $\delta \hat{R}_{g}$ and $\delta \hat{C}_{g}$ cannot be considered as representing symmetrical changes in airway properties. Instead, taking into account that spirometry is insensitive to airway inhomogeneities [24], they should be interpreted as a measure of effective changes in subsequent airway generations.

The accuracy of InvM parameter estimates strongly depends on the quality of global identification done using the InvNN [29], and thus the effect of InvNN influences also the ultimate results of the proposed method. This is because $\hat{\boldsymbol{\theta}}_{\text {InvM }}^{(6)}$ are kept unchanged during the estimation of MAR parameters, so their better evaluation in relation to the true values would improves the reduction of systematic errors in this differential measurement (see Apendix). The InvNN has been trained with synthetic data assuming that the properties of lungs with dilated airways are similar to the healthy state, which need not be the case. The new estimates of InvM parameters gathered in this work by the analysis of real post-test data can be used to develop an InvNN more adequate for asthmatic patients in the future, so it should further improve the accuracy of the method.

Predictably, the best accuracy was achieved when analyzing spirometry data generated by the InvM (Table I and Fig. 5), since all the parameters, except the 6 estimated, had the same values during simulation and identification, and the systematic errors were negligible (as follows from the Appendix). In this case, the RMSE were dominated by random distribution of estimates, stemming mainly from the imperfect results of global identification by the InvNN and random noise added to the flow data [29]. Releasing the values of constant parameters during data generation with the SymM yielded a bit worse results, proving that their impact on the results is small, and that the use of InvM with the 6 degrees of freedom is justified. The results obtained with the synthetic data generated by the most reliable AsymM are particularly interesting. Especially useful is the evaluation of $\delta R_{g}$ in the zone of conducting airways, because this segment makes the main contribution to total airway resistance, affecting breathing effort. On the other hand, the quantitative insight into changes in the acinar airways is less accurate. It is not surprising, because it is well known that spirometry is little sensitive to the processes in this region, called the "silent zone" [25], [34], [39]. It should be also noted that the worse results obtained for data from the AsymM, particularly the errors of $p_{g}, p_{w}$ and $\delta C_{g}$ estimation, may be caused by the linear transformation between the Horsfield and Weibel geometries of the bronchial tree, applied to translate the true airway properties that characterized 31 orders, into 24 generations used in the InvM and MAR. Such a transformation is not obvious, because in each generation there are airways of a few orders, as shown in [35]. This is why the assessment of $\hat{p}_{s}, \hat{p}_{g}$ and $\hat{p}_{w}$ accuracy obtained from the SymM data seems to be more reliable for the MAR parameters, together with the percentage of correctly indicated airway orders with the maximal response, calculated based on the AsymM data, which exceeds $91 \%$ in the case of conducting bronchi. The differences 
between the estimated and true airway responses to bronchodilation have not been analyzed between the mouth and the flow-limiting sites, because the descending parts of spirometric curves are independent from the airway properties downstream these points [36], and the estimates describing this region are unreliable.

The potential of the proposed method is shown on the example of bronchodilator test data from three asthmatic patients. Averaging the three pre- and post-bronchodilator expiratory flows reduced random fluctuation visible in the individual curves, which should have a positive impact on the accuracy of estimation (Fig. 6). From the decreased resistances and increased compliances (Fig. 7), it is apparent that ASM has reduced its tension after inhalation of salbutamol, and the airways have effectively dilated. According to the obtained results, the sites of airway response to the administrated medication were located approximately between generation 4 and 12 for Patients \#1 and \#3, with maximum around generation 8, and between generations 9 and 13 (maximum at 11-12) for Patient \#2. This is in agreement with anatomical and functional studies on beta-adrenoceptors in human lung [6], [37], [38], [39]. Using the results of the method accuracy analysis (Fig. 5) for Patient \#2 as an example (Fig. 7), it can be stated that the maximal drop of airway resistance was $55-73 \%$, and the maximal increase of compliance was $50-78 \%$. It is also worth noting that the analyzed cases were not homogeneous regarding their age and height, which may explain the clustered results (Patients \#1 and \#3 were young and tall, whereas Patient \#2 was older and shorter). It is well-known that the processes underlying airway obstruction develop with aging, and are responsible for pulmonary function decline and changes in airway responsiveness [40].

\section{CONCLUSION}

The method for quantitative assessment of the airway response to bronchial tests deduced from the shift of the spirometric curve, in terms of the degree, generation with maximal response, and width of changes along the bronchial tree that can be recalculated into relative changes in airway resistances and compliances, is prosed in this work. Such an approach to processing spirometry data into respiratory properties of clinical meaning has not been developed so far.

The evaluation of method accuracy has been done using synthetic data generated by the most reliable computational models for forced expiration currently available. The results of analyses show that the assessment of the site and degree of changes is plausible, particularly in the zone of conducting airways. Thus, the proposed method may be used not only to infer about the clinical effects of bronchial tests, but also to monitor changes in the airways between ambulatory visits or to investigate how known or new pharmaceuticals affect the bronchi.

As the future work, an experimental validation of the method by comparing its outcomes with the results of high-resolution computed tomography performed synchronously with the bronchial tests is planned [41], [42], as well as clinical trials with a larger group of subjects (preferably suffering from asthma and COPD) and different drugs inhaled.

\section{APPENDIX}

The benefits of using differential measurements can be easy clarified with a simple example. Suppose a model $f$ with the output $y_{m}$, excited by the known input $u$, has two parameters, $\alpha$ and $\beta: y_{m}=f(\alpha, \beta, u)$. Then the idea of indirect measurement of $\alpha$ can be formally expressed as:

$\alpha=f_{\alpha}^{-1}\left(\beta, u, y_{m}\right)=g\left(\beta, u, y_{m}\right)$

where $f_{\alpha}^{-1}=g$ is the inverse mapping with respect to $\alpha$ (usually impossible to find analytically, so most inverse problems are solved numerically). To calculate the true value $\alpha^{*}$, one has to know the true $\beta^{*}$ and $u$, and measured $y_{m}$ (random measurement errors are ignored here). In the case under consideration, the value $\tilde{\beta}$ is however uncertain, and thus is also $\tilde{\alpha}$. The relationship between $\tilde{\alpha}$ and $\tilde{\beta}$ can be expressed by the Taylor series:

$\tilde{\alpha}=g\left(\tilde{\beta}, u, y_{m}\right)=\alpha^{*}+\sum_{n=1}^{\infty} \frac{1}{n !}\left(\frac{\partial^{n} g}{\partial \beta^{n}}\right)^{*}\left(\tilde{\beta}-\beta^{*}\right)^{n}$

where $\alpha^{*}=g\left(\beta^{*}, u, y_{m}\right)$. For models linear in parameters, $(\partial g / \partial \beta)^{*}$ is a constant $c_{1}$, and $\left(\partial^{n} g / \partial \beta^{n}\right)^{*}=0$ for $n>1$, so having two $y_{m}$ for unknown but modified $\alpha_{1}^{*}$ and $\alpha_{2}{ }_{2}$, the difference between the uncertain results of indirect measurements:

$\tilde{\alpha}_{2}-\tilde{\alpha}_{1}=\left(\alpha_{2}^{*}+c_{1} \Delta \beta\right)-\left(\alpha_{1}^{*}+c_{1} \Delta \beta\right)=\alpha_{2}^{*}-\alpha_{1}^{*}$

where $\Delta \beta=\tilde{\beta}-\beta^{*}$, is theoretically correct, because the systematic errors cancel each other. For nonlinear models, however, the values of partial derivatives $c_{n}$ at the point of Taylor's expansion depend on $\alpha^{*}$, so the systematic error of differential measurement does not disappear, but is reduced:

$\tilde{\alpha}_{2}-\tilde{\alpha}_{1}=\alpha_{2}^{*}-\alpha_{1}^{*}+\sum_{n=1}^{\infty} \frac{1}{n !}\left[c_{n}\left(\alpha_{2}^{*}\right)-c_{n}\left(\alpha_{1}^{*}\right)\right](\Delta \beta)^{n}$

Summarizing, in the case of nonlinear models, the accuracy of differential measurement is higher for similar nonlinearity effects $c_{n}\left(\alpha_{1}^{*}\right)$ and $c_{n}\left(\alpha_{2}^{*}\right)$ (their difference is small for a slight alteration from $\alpha_{1}^{*}$ to $\alpha_{2}^{*}$ ), as well for better evaluation of $\tilde{\beta}$. In the context of this work, were $\alpha$ is the equivalent of airway properties, $\beta$ represents both the parameters estimated by InvM and then used in MAR and all other parameters kept constant in the models, the principle of differential measurement was applied to estimate $p_{s}, p_{g}$ and $p_{w}$, and then to calculate the changes in the airway generation resistance and compliances in (5). 


\section{REFERENCES}

[1] R. Pellegrino et al., "Interpretative strategies for lung function tests," Eur. Respir. J., vol. 26, no. 5, pp. 948-968, Nov. 2005.

[2] Anonymous. (2019). Global strategy for asthma management and prevention. Global Initiative for Asthma, WI. [PDF]. Available: www.ginasthma.org

[3] Anonymous. (2020). Global strategy for prevention, diagnosis and management of COPD. Global Initiative for Chronic Obstructive Lung Disease, WI. [PDF]. Available: www.goldcopd.org

[4] B. J. Lipworth and D. G. McDevitt, "Beta-adrenoceptor responses to inhaled salbutamol in normal subjects," Eur. J. Clin. Pharmacol., vol. 36, no. 3, pp. 239-245, 1989.

[5] M. R. Miller, et al., "Standardisation of spirometry," Eur. Respir. J., vol. 26, no. 2, pp. 319-338, Aug. 2005.

[6] J. R. Carstairs et al., "Autoradiographic visualization of betaadrenoceptors in human lung," Am. Rev. Respir. Dis., vol. 132, no. 3, pp. 541-547, Sep. 1985.

[7] P. G. Koullapis et al., "Particle deposition in a realistic geometry of the human conducting airways: Effects of inlet velocity profile, inhalation flowrate and electrostatic charge," J. Biomech., vol. 49, no. 11, pp. 22012212, Jul. 2016.

[8] M. S. Islam et al., "Ultrafine particle transport and deposition in a large scale 17-generation lung model," J. Biomech., vol. 64, pp. 16-25, Nov. 2017.

[9] A. V. Kolanjiyil and C. Kleinstreuer, "Computational analysis of aerosoldynamics in a human whole-lung airway model," J. Aerosol Sci., vol. 114, pp. 301-316, Oct. 2017.

[10] Q. Deng et al., "Particle deposition in tracheobronchial airways of an infant, child and adult," Sci. Total Environ., vol. 612, pp. 339-346, Jan. 2018.

[11] C. Darquenne and G. K. Prisk, "The effect of aging on aerosol bolus deposition in the healthy adult lung: A 19 -year longitudinal study," $J$. Aerosol Med. Pulm. Drug Delivery, DOI: 10.1089/jamp.2019.1566, Oct. 2019.

[12] J. Dong et al., "Ultrafine particle deposition in a realistic human airway at multiple inhalation scenarios," Int. J. Numer. Methods Biomed. Eng., vol. 35, no. 7, e3215, Jul. 2019.

[13] N. Scichilone et al., "Assessing and accessing the small airways; implications for asthma management," Pulm. Pharmacol. Ther., vol. 26, no. 2, pp. 172-179, Apr. 2013.

[14] S. Ostadabbas et al., "A vision-based respiration monitoring system for passive airway resistance estimation," IEEE Trans. Biomed. Eng., vol. 63, no. 9, pp. 1904-1913, Sep. 2016.

[15] D. K. Walters et al., "Efficient, physiologically realistic lung airflow simulations," IEEE Trans. Biomed. Eng., vol. 58, no. 10, pp. 3016-3019, Oct. 2011.

[16] A. Beaulieu et al., "Measurement of fractional order model parameters of respiratory mechanical impedance in total liquid ventilation," IEEE Trans. Biomed. Eng., vol. 59, no. 2, pp. 323-331, Feb. 2011.

[17] C. M. Ionescu et al., "A recurrent parameter model to characterize the high-frequency range of respiratory impedance in healthy subjects," IEEE Trans. Biomed. Circ. Syst., vol. 7, no. 6, pp. 882-892, Dec. 2013.

[18] J. Glapiński et al., "Analysis of the method for ventilation heterogeneity assessment using the Otis model and forced oscillations," Comput. Methods Programs Biomed., vol. 122, no. 3, pp. 330-340, Dec. 2015.

[19] R. Amini et al., "Intratidal overdistention and derecruitment in the injured lung: a simulation study," IEEE Trans. Biomed. Eng., vol. 64, no. 3, pp. 681-689, Mar. 2017.

[20] C. J. Roth et al., "A comprehensive computational human lung model incorporating inter-acinar dependencies: Application to spontaneous breathing and mechanical ventilation," Int. J. Numer. Meth. Biomed. Eng., vol. 33, no. 1, e02787. Jan. 2017.

[21] R. K. Lambert et al., "A computational model for expiratory flow," $J$. Appl. Physiol.: Respirat. Environ. Exercise Physiol., vol. 52, no. 1, 4456, Jan. 1982.
[22] A. G. Polak, "A forward model for maximum expiration," Comput. Biol. Med., vol. 28, no. 6, pp. 613-625, Nov. 1998.

[23] K. L. Hedges and M. H. Tawhai, "Simulation of forced expiration in a biophysical model, with homogeneous and clustered bronchoconstriction," J. Biomech. Eng., vol. 138, no. 6, 061008, Jun. 2016.

[24] A. G. Polak et al., "Effects of homogeneous and heterogeneous changes in the lung periphery on spirometry results," Comput. Methods Programs Biomed., vol. 173, pp. 139-145, May 2019.

[25] R. K. Lambert, "Sensitivity and specificity of the computational model for maximal expiratory flow," J. Appl. Physiol., vol. 57, no. 4, pp. 958970, Oct. 1984.

[26] R. K. Lambert et al., "Model of forced expiratory flows and airway geometry in infants," J. Appl. Physiol., vol. 96, no. 2, pp. 688-692, Feb. 2004.

[27] R. K. Lambert and K. C. Beck, "Airway area distribution from the forced expiration maneuver," J. Appl. Physiol., vol. 97, no. 2, pp. 570-578, Aug. 2004

[28] B. Morlion and A. G. Polak "Simulation of lung function evolution after heart-lung transplantation using a numerical model," IEEE Trans. Biomed. Eng., vol. 52, no. 77, pp. 1180-1187, Jul. 2005.

[29] A. G. Polak et al., "Estimation of lung properties from the forced expiration data," IEEE Trans. Instrum. Meas., vol. 69, no. 6, pp. 33173324, Jun. 2020.

[30] A. G. Polak et al., "Analysis of the method for determining changes in the airways from the spirometric curve evolution," in ACM Proceedings of the 8th International Conference on Bioinformatics and Biomedical Science, Beijing, China, 2019, pp. 120-125.

[31] C. E. Chávez et al., "Avoiding the inverse crime in the inverse problem of electrocardiography: estimating the shape and location of cardiac ischemia," in: IEEE Comput. Cardiol., 2013, pp. 687-690.

[32] A. G. Polak, "A model-based method for flow limitation analysis in the heterogeneous human lung," Comput. Methods Programs Biomed., vol. 89, no. 2, pp. 123131, Feb. 2008.

[33] T. Chai and R. R. Draxler, "Root mean square error (RMSE) or mean absolute error (MAE)? - Arguments against avoiding RMSE in the literature," Geosci. Model Dev., vol. 7, no. 3, pp. 1247-1250, Jun. 2014.

[34] J. Mead, "The lung's quiet zone," N. Engl. J. Med., vol. 282, no. 23, pp. 1318-1319, Jun. 1970.

[35] B. R. Wiggs et al., "A model of the mechanics of airway narrowing," $J$. Appl. Physiol., vol. 69, no. 3, pp. 849-860, Sep. 1990.

[36] P. J. G. M. Voets and H. A. C. van Helvoort, "The role of equal pressure points in understanding pulmonary diseases," Adv. Physiol. Educ., vol. 37, no. 3, pp. 266-267, Sep. 2013.

[37] L. A. De Backer et al., "Functional imaging using computer methods to compare the effect of salbutamol and ipratropium bromide in patientspecific airway models of COPD," Int. J. Chron. Obstruct. Pulmon. Dis., vol. 6, pp. 637-646, Nov. 2011

[38] S. J. Park et al., "Quantitative analysis of dynamic airway changes after methacholine and salbutamol inhalation on xenon-enhanced chest CT," Eur. Radiol., vol. 22, no. 11, pp. 2441-2450, Nov. 2012.

[39] Z. Su et al. (2020, Jan.). Airway remodeling and bronchodilator responses in asthma accessed by endobronchial optical coherence tomography. [Abstract online]. Available: https://ssrn.com/abstract=3516151

[40] A. Bush, "Lung development and aging," Ann. Am. Thorac. Soc., vol. 13, suppl. 5, pp. S438-S446, Dec. 2016.

[41] A. Obojski et al., "Quantitative computed tomography imaging in asthma and chronic obstructive pulmonary disease," Eur. Resp. J., vol. 52, suppl. 62, OA3796, Nov. 2018.

[42] M., Patyk et al., "Airway wall thickness and airflow limitations in asthma assessed in quantitative computed tomography," Ther. Adv. Respir. Dis., vol. 14, 1753466619898598, 2020.

[43] G. G. King et al., "Pathophysiology of severe asthma: We've only just started," Respirology, vol. 23, no. 3, pp. 262-271. Mar. 2018.

[44] N. Tanabe et al., "Analysis of airway pathology in COPD using a combination of computed tomography, micro-computed tomography and histology," Eur. Respir. J., vol. 51, no. 2, 1701245, Feb. 2018. 